\title{
Improving Interaction with Services via Probabilistic Piggybacking*
}

\author{
Carlo Ghezzi ${ }^{1}$, Mauro Pezzè ${ }^{2}$, and Giordano Tamburrelli ${ }^{2}$ \\ 1 Dipartimento di Elettronica e Informazione. Politecnico di Milano, Italy \\ carlo.ghezzi@polimi.it \\ 2 Faculty of Informatics. University of Lugano, Switzerland \\ \{mauro.pezze, giordano.tamburrelli\}@usi.ch
}

\begin{abstract}
Modern service oriented applications increasingly include publicly released services that impose novel and compelling requirements in terms of scalability and support to clients with limited capabilities such as mobile applications. To meet these requirements, service oriented applications require a careful optimisation of their provisioning mechanisms. In this paper we investigate a novel technique that optimises the interactions between providers and clients called probabilistic piggybacking. In our approach we automatically infer a probabilistic model that captures the behaviour of clients and predicts the future service requests. The provider exploits this information by piggybacking each message toward clients with the response of the predicted next request, minimizing both the amount of exchanged messages and the client latency. The paper focuses on REST services and illustrates the technique with a case study based on a publicly available service currently in use.
\end{abstract}

\section{Introduction}

Service oriented applications (SOC) [16] support the integration and collaboration among internal entities of the same organisation and enable industrial partnerships across distinct organisations, independently from their technological stacks. Recently, software services are increasingly released to the general public allowing independent developers and external software organisations to create new software artifacts that leverage them.

The increasing popularity of openly accessible services introduces novel software engineering challenges that affect the way clients exploit services as well as the mechanisms adopted by service providers to offer them. From the service provider perspective, SOC applications need to meet new compelling requirements in terms of scalability, being concurrently accessed by large and unpredictable populations of distinct clients. Indeed, the massive stream of requests that service providers have to deal with may hamper their capability to operate efficiently,

\footnotetext{
* This research has been funded by the EU, Programme IDEAS-ERC, Project 227977SMScom and FP7-PEOPLE-2011-IEF, Project 302648-RunMore.
}

S. Basu et al. (Eds.): ICSOC 2013, LNCS 8274, pp. 39-53, 2013.

(C) Springer-Verlag Berlin Heidelberg 2013 
potentially violating existing service level agreements. For example, the Twitter REST service 1 serve several billions of requests per day and several thousands calls per second. Moreover, from the client perspective, the interactions with the service providers need to be as efficient as possible for example in terms of number of exchanged messages and latency. Indeed, an increasing percentage of existing clients is represented by mobile applications that are typically characterised by limited bandwidth, unreliable connectivity, and limited computational power. To effectively support the required scalability and to optimise the interactions with clients characterised by limited capabilities, modern SOC applications require efficient service provisioning mechanisms. This paper addresses this issue and introduces a technique that focuses on REST services [822] called probabilistic piggybacking.

Probabilistic piggybacking consists of an inference algorithm that monitors at runtime all the requests issued by a client and incrementally infers a probabilistic model of its behaviour in terms of service invocations. Given a certain service invocation currently issued by a client, the inferred probabilistic model predicts the next service invocation it may issue next. The service provider exploits this information by piggybacking each message towards the client with the response to the predicted request. Probabilistic piggybacking brings two distinct advantages, (1) reduces client latency since responses to predicted invocations are immediately available to the client, and (2) optimises the interaction among clients and service providers since each correctly predicted invocation reduces the number of exchanged messages. In addition, as discussed later on in Section 5 . the proposed solution is transparent to the client, requires a minimum modification to the server implementation, and is complementary to other existing related techniques such as caching.

This paper contributes to current research in service engineering in two distinct ways. First, it defines an inference algorithm for SOC applications that captures the usage profiles of clients and predicts their service invocation patterns. Second, it proposes a novel probabilistic piggybacking technique based on the inference algorithm to optimise REST service provisioning mechanisms. The inference algorithm and the proposed technique have been validated with experiments and simulations extracted from a case study of a set of REST services currently in use.

The remainder of the paper is organized as follows. Section 2 overviews the probabilistic piggybacking technique. Section 3 introduces the running case study, namely a set of REST services publicly available online and currently in use that we use throughout the paper to exemplify and validate the approach. Section 4 provides a detailed description of the approach through the case study, while Section 5 presents the results of our analytical and experimental evaluation of the proposed approach and its applicability. Section 6 discusses the related work. Section 7 summarizes the main contributions of the paper, and illustrates the ongoing research work.

${ }^{1}$ https://dev.twitter.com 


\section{Probabilistic Piggybacking: An Overview}

Piggybacking [17] is a well-known data transmission technique that belongs to the network layer and improves transmission efficiency. In this technique, the acknowledgements to data received from an emitter by a sender are attached to the messages emitted in the opposite direction. More precisely, piggybacking implies that an acknowledgement sent by a receiver, instead of being sent in an individual message, is piggybacked on a data frame going toward the sender. In this paper we conceived a probabilistic technique inspired by the basic principle of piggybacking that optimises service provisioning. Although the proposed technique is in principle applicable to many different SOC styles, we refer to services implemented according to a REST architectural style. REST is an increasingly popular 2 architectural style in which requests and responses flow through the stateless transfer of resources via http urls that uniquely identify the state of the conversation between clients and servers. A complete discussion of the REST architectural style is beyond the scope of this paper, the interested readers can find additional details in 822 .

The probabilistic piggybacking technique includes two fundamental components: (1) the inference engine and (2) the piggybacking engine. The former is deployed to the client, while the latter is deployed to the service provider. The technique is articulated in four main steps that we introduce below. The first step occurs at design time, while the others take place at runtime.

1. Specifying Endpoints: At design time providers specify the service endpoints using a lightweight service description language. This description integrates the service documentation and is publicly released to clients.

2. Inferring the model: The inference engine monitors the service requests issued by a client and infers a discrete time Markov chain (DTMC) [18. DTMCs are finite state automata augmented with probabilities. The Markov model is built incrementally as soon as new requests are issued to the service provider.

3. Predicting requests: The inference engine uses the inferred model to predict the next most likely service invocation, given a currently issued service request. The prediction is sent, together with the request currently issued, to the provider.

4. Instantiating request parameters: The service provider serves the requests as soon as they are issued and receives with each of them the endpoint predicted by the inference engine deployed on the client side. The piggybacking engine - deployed on the service provider - exploits this information, instantiates the actual parameters of the predicted endpoint, and prepares the corresponding response to be sent to the client via piggybacking (i.e., attached to the response of the request issued by the client). The response piggybacked to the client is cached. If the prediction made by the inference engine is correct the cached response will be immediately available thus reducing the latency and avoiding otherwise the client to issue a new service invocation to the provider.

\footnotetext{
${ }^{2}$ According to ProgrammableWeb, the largest directory of publicly available services, the percentage of publicly available REST services listed in their platform is gradually increasing and recently reached $68 \%$. http://www.programmableweb.com
} 
Table 1. Flixster Movie REST Api

\begin{tabular}{|c|c|c|c|c|}
\hline Name & Type & Service Endpoint & Description & Url Parameter (default value) \\
\hline search & GET & /movies.json & $\begin{array}{l}\text { The movies search } \\
\text { endpoint for plain } \\
\text { text queries }\end{array}$ & $\begin{array}{l}\text { q query to search (-) } \\
\text { page_limit results per page }(30) \\
\text { page selected page }(1)\end{array}$ \\
\hline info & GET & /movies/〈id>.json & $\begin{array}{c}\text { Detailed information } \\
\text { on a specific movie } \\
\text { specified by the }\langle\text { id }\rangle\end{array}$ & - \\
\hline cast & GET & $/$ movies/<id>/cast.json & $\begin{array}{l}\text { Retrieves the complete } \\
\text { cast for a movie } \\
\text { specified by the }\langle i d\rangle\end{array}$ & - \\
\hline clips & GET & /movies/<id>/clips.json & $\begin{array}{l}\text { Retrieves the trailer } \\
\text { for a movie } \\
\text { specified by the }\langle\text { id }\rangle\end{array}$ & - \\
\hline similar & GET & /movies/<id>/similar.json & $\begin{array}{c}\text { Retrieves similar } \\
\text { movies for a movie } \\
\text { specified by the }\langle\text { id }\rangle\end{array}$ & limit max number of results (5) \\
\hline
\end{tabular}

\section{Motivating Example}

In this section we introduce an example of popular REST services that we use through the paper to illustrate and motivate our research. The case study refers to the Flixster movie REST ap 3 that gives access to the Flixster movie database, allowing independent developers or external organisations to build applications and widgets containing movies data. Using these api users can search for movies, retrieve detailed movie information like cast or directors, and access movie trailers. The Flixster movie REST api includes other endpoints (related to upcoming movies, dvd, etc) that we omit due to the limited space. Service endpoints return responses in the $\mathrm{JSON}_{4}$ standard format: a lightweight data-interchange format alternative to XML increasingly popular for REST services. Table 1 lists the service endpoints with a brief textual description. The proposed solution focuses on optimising GET requests and ignores other kinds of messages (e.g., POST). Indeed, GET requests correspond to requesting specific resources and retrieving data without any side-effect on the server [7. In other words GET requests are idempotent read requests that can be predicted and served by our piggybacking mechanism without hampering the semantics of the interaction between client and server. In addition, GET requests represent, in practice, the most frequent class of messages exchanged among clients and REST service providers.

In REST services, GET requests may have two distinct classes of parameters: structural parameters and url parameters. Structural parameters refer to parametric fragments of the path of a certain endpoint used to customise requests issued by the clients. For example, clients parametrize the path of the similar service endpoint with respect to the id of the movie they are interested in. Url parameters refer to parameters appended at the end of the endpoint in the form name=value and interleaved by an ampersand. For example, the similar service endpoint may be parametrized with the limit url parameter as reported in the

3 http://developer.rottentomatoes.com

4 http://www.json.org 


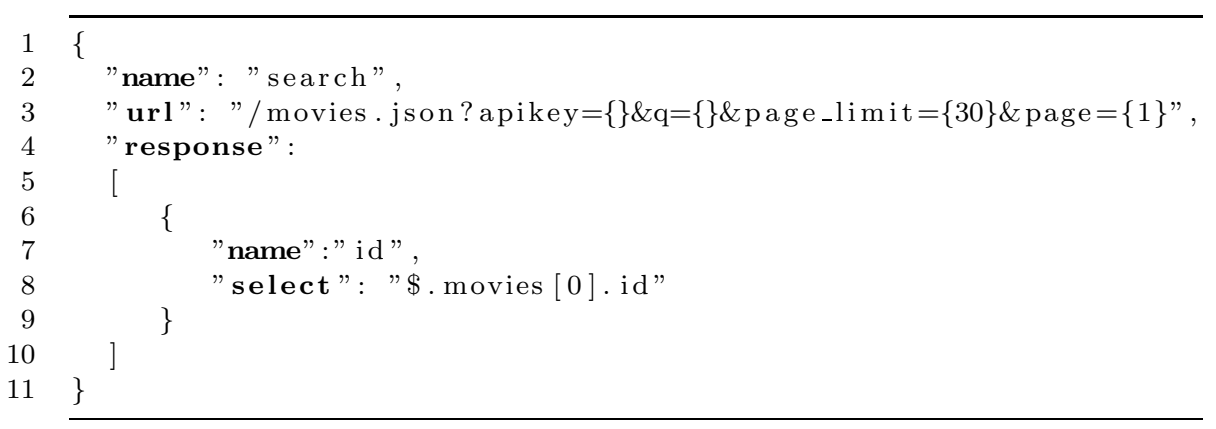

Listing 1.1. RDL endpoint definition with response field

fourth column of Table 1. The fourth column of the table reports the default value of url parameters (i.e., the value of the parameter if omitted in the request). All the endpoints reported in Table 1 require the additional mandatory url parameter apikey used by the service provider to identify the clients. The following url is an example of a valid service request to the similar endpoint:

$$
\text { /movies/77/similar.json?apikey=798\&limit=30 }
$$

In this case the client has an apikey equal to 798, the movie targeted by the request has an id equal to 77 , and the request will return at most 30 movies.

As introduced in Section 1, thousands of clients may simultaneously access these endpoints and an increasing number of these accesses is performed by client with limited capabilities such as mobile applications. As a consequence, the service provisioning mechanisms should be as efficient and scalable as possible.

\section{Probabilistic Piggybacking Explained}

In this section we explain in details the probabilistic piggybacking approach and how it optimises the service provisioning mechanisms referring to the four steps previously introduced and to the Flixster movie services.

\subsection{Specifying Endpoints}

Providers describe the endpoints with a lightweight service description language in the JSON format called RDL5. We conceived RDL specifically for probabilistic piggybacking, not with the goal of designing a new service specification language that could replace existing standards, such as WSDL [5. Indeed, the scope of RDL is limited to supporting the piggybacking operations and not to specifying or documenting the endpoints.

${ }^{5} \underline{R} E S T \underline{\text { Description L Language }}$ 
The RDL service description consists of a list of JSON objects. Each of them contains the description of an endpoint in terms of its name and its url. The url representation includes its parameters as follows. Structural parameters in the url are specified by a name within curly brackets (\{parameter_name $\}$ ), while url parameters are specified by their name followed by an equal sign and curly brackets that may contain the default value of the parameter (parameter_name $=\{$ default_value $\}$ ). As an example, let us consider Listing 1.1, which shows the RDL representation of the Flixster search endpoint. The response field in the RDL description may be used to select a string fragment of the endpoint response and to assign to it a name. For this task RDL relies on a JSONPath 6 expression. JSONPath is a path expression syntax, which can select parts of JSON documents in the same way as XPath [1] expressions select nodes of XML documents. For example, in the case of the search endpoint specified in Listing 1.1. we may want to select the id of the first movie in the response by labelling it with the string " $i d$ ". The index 0 indicates we are interested in the first item of the array of the movies returned in the response. We clarify the meaning of an RDL response field in the context of probabilistic piggybacking in Section 4.4 .

The provider of Flixster movie services may easily translate all the endpoints illustrated in Table 1 to RDL obtaining a complete description to be publicly released to the clients.

\subsection{Inferring the Model}

Clients invoke service endpoints by issuing http requests to the endpoint urls. In our approach, each request issued by a client is intercepted by the inference engine and used to incrementally infer a discrete time Markov chain (DTMC) [18] used to predict the next request by the client. DTMCs are finite state automata augmented with probabilities. In our setting each state corresponds to a service endpoint, while probabilistic transitions among states represent the probabilities of sequences of invocations. Formally:

A DTMC is a tuple $\left\langle\mathcal{S}, s_{0}, \mathcal{P}, \mathcal{N}, \mathcal{L}\right\rangle$ where:

$\mathcal{S}$ : is a non empty finite set of states, where $s_{0} \in S$ is the initial state;

$\mathcal{P}: \mathcal{S} \times \mathcal{S} \rightarrow[0,1]$ is a stochastic function representing the probabilistic edges that connect the states in $\mathcal{S}$. An element $\mathcal{P}\left(s_{i}, s_{j}\right)$ represents the probability that the next state will be $s_{j}$ given that the current state is $s_{i}$;

$\mathcal{N}$ : is the set of endpoint names extracted from the RDL file;

$\mathcal{L}: \mathcal{S} \rightarrow \mathcal{N}$ is a labeling function associating each state to an endpoint name;

The inference engine uses the RDL specification of endpoints to identify and label states. The inference process starts from a DTMC with a set of states $\mathcal{S}$ that contains only the initial state $s_{0}$ and the initial labelling function $\mathcal{L}\left(s_{0}\right)=$ start. The inference engine builds the DTMC incrementally by processing the service requests as they are issued by the client adding new states to DTMC and inferring transitions between states in $\mathcal{S}$. For each request the inference engine

\footnotetext{
6 http://goessner.net/articles/JsonPath/
} 


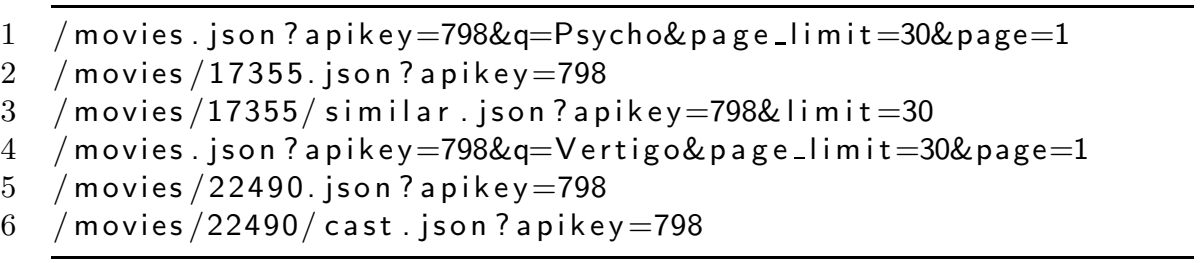

Listing 1.2. A list of REST requests

infers a transition following a 3-step process: extracting the destination state, extracting the source state, and computing the probabilities.

1. Extracting the destination state. The inference engine examines the current request $r$ and maps the request to its corresponding endpoint name $n$ exploiting the RDL file. Thus the engine associates a destination state $d \in \mathcal{S}$ to $r$ such that $\mathcal{L}(d)=n$. If $d$ does not belong to $\mathcal{S}$ yet, the inference engine adds the new state $d$ to $\mathcal{S}$ and updates the labelling function $\mathcal{L}$ accordingly.

2. Extracting the source state. The engine associates the destination state of the last invoked service request to the source state of $r$ and associates the first service request issued by the client to the initial state $s_{0}$.

3. Computing the probabilities. The engine uses the intercepted service invocations to update two sets of counters that are initially set to zero: a set of counters $c_{i, j}$ for each pair of states $\left(s_{i}, s_{j}\right) \in \mathcal{S} \times \mathcal{S}$, and a set of counters $t_{i}$ for each state $s_{i} \in S$. The inference engine increments both the counter $c_{i, j}$ for each transition from state $s_{i}$ to $s_{j}$ and the counter $t_{i}$ for each transition whose source state is $s_{i}$, independent of its destination state. The counter $t_{i}$ represents the number of times the clients exited state $s_{i}$, while the counter $c_{i, j}$ represents the number of times the clients moved from state $s_{i}$ to state $s_{j}$. The inference engine updates the counters for each request that corresponds to a transition in the model, and uses these counters to calculate the $(i, j)$ entry of the stochastic function $\mathcal{P}$ that represents the probability of traversing the edge from state $s_{i}$ to state $s_{j}$, by computing the following frequency:

$$
\mathcal{P}\left(s_{i}, s_{j}\right)=\frac{c_{i, j}}{t_{i}}
$$

for all pairs of states $s_{i}$ and $s_{j}$. The probability $\mathcal{P}\left(s_{i}, s_{j}\right)$ computed as the ratio between the number of traversals of the transition from the state $s_{i}$ to $s_{j}$ and the total number of traversals for all the transitions exiting state $s_{i}$ corresponds to the maximum likelihood estimator for $\mathcal{P}\left(s_{i}, s_{j}\right)$ [6. Notice that the probabilities can be recomputed incrementally after adding any number of transitions or states to the DTMC.

Figure 1 illustrates the inference process described so far, by referring to the sequence of invocations of a given client reported in Listing 1.2. We start from 


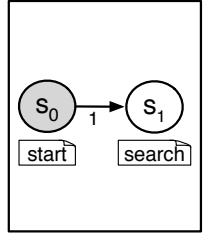

(a)

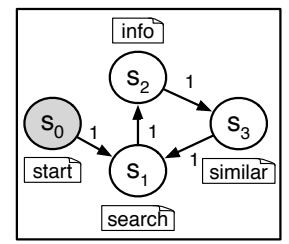

(b)

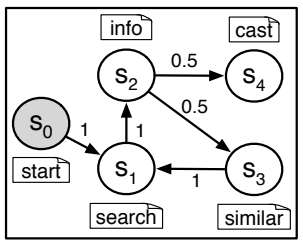

(c)

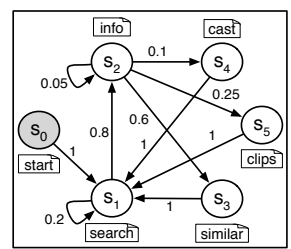

(d)

Fig. 1. DTMC inference process

the initial DTMC shown in Figure 1(a) and proceed incrementally through the invocations. The first invocation is associated with the endpoint search. Since $\mathcal{S}$ does not contain any state $s$ such that $\mathcal{L}(s)=$ search, the engine adds a new state $s_{1}$ to $\mathcal{S}$, and extends the labelling function with $\mathcal{L}\left(s_{1}\right)=$ search. Being this the first service invocation of the client, the engine considers state $s_{0}$ as the source state for the inferred transition $\left(\left\langle s_{0}, s_{1}\right\rangle\right)$. The engine increments the counters $t_{0}$ and $c_{0,1}$, and consequently $\mathcal{P}\left(s_{0}, s_{1}\right)$ is set to 1 . The resulting DTMC is shown in Figure 1(a). The second request corresponds to a request of detailed information on a specific movie with id equal to 17355 and is associated with the endpoint info. The inference engine associates the new destination state $s_{2}$ to this request and updates the labelling function: $\mathcal{L}\left(s_{2}\right)=$ info. The engine identifies the destination state of the last invoked service request as the source state yielding to a transition $\left\langle s_{1}, s_{2}\right\rangle$. The engine increments the counters related to the new transition: $t_{1}=1$ and $c_{1,2}=1$. Consequently, $\mathcal{P}\left(s_{1}, s_{2}\right)$ is set to 1 .

Similarly, the third request is associated with a new destination state $s_{3}$ such that $\mathcal{L}\left(s_{3}\right)=$ similar. The source state is associated again with the destination state of the last transition generated $\left(s_{2}\right)$ and results in transition $\left\langle s_{2}, s_{3}\right\rangle$. The engine increments the counters $t_{2}$ and $c_{2,3}$, and consequently sets $\mathcal{P}\left(s_{2}, s_{3}\right)=1$. The fourth request results in the transition $\left\langle s_{3}, s_{1}\right\rangle$. The resulting DTMC is reported in Figure 1(b), The fifth request represents again a request to the info endpoint and is treated as previously shown. The sixth request infers a new destination state $s_{4}$, where $\mathcal{L}\left(s_{4}\right)=$ cast, and a new transition $\left\langle s_{2}, s_{4}\right\rangle$. The engine increments the counters $t_{2}$ and $c_{2,4}$, and consequently sets $\mathcal{P}\left(s_{2}, s_{4}\right)=0.5$ and $\mathcal{P}\left(s_{2}, s_{3}\right)=0.5$. Figure $1(\mathrm{c})$ shows the resulting DTMC.

By applying the inference algorithm to the requests issued by a client we eventually obtain a DTMC that probabilistically captures its usage profile. For example, if a client issued 350 requests to the search endpoint and 280 of these requests were followed by a request to the info endpoint, in the inferred DTMC the transition $\left\langle s_{1}, s_{2}\right\rangle$ would be associated with probability 0.8 . An example of a possible inferred DTMC for the Flixster services is shown in Figure $1(\mathrm{~d})$.

In this paper we refer to client applications that deal with only one service provider. This is not always the case if we consider for example applications that organise and exploit multiple services such as service mashups or service compositions. The proposed technique is applicable seamlessly also to these 
multi-provider examples by simply instantiating several inference engines, one for each provider, that independently infer distinct client behaviours.

\subsection{Predicting Requests}

The inference engine intercepts all the outgoing service requests issued by the client to infer the DTMC model as explained above. At each intercepted service request, the engine uses the inferred model to predict the service endpoint that the client will invoke in the next service request.

Given a request $r$ to a certain service endpoint $e$, the engine analyzes the state in the DTMC representing $e$ and selects the outgoing transition with the highest probability. If the transition probability is greater than a given threshold (the piggybacking_threshold) the engine considers its destination state as a valuable prediction of the most likely next service request. The engine communicates this information to the server by appending an additional url parameter (called piggyback) to the outgoing request $r$ that indicates the predicted endpoint name as specified in the RDL file. If the transition probability is less than the piggybacking_threshold, the engine does not append the additional parameter and the probabilistic piggybacking simply does not occur for this request.

Let us consider the case where a client issued a request to the info endpoint for the movie with id equal to 77 . In the scenario in which the DTMC indicates that the most likely service endpoint invoked next is the similar endpoint, the url actually issued by the client is the following:

$$
\text { /movies/77.json?apikey=798\&piggyback=similar }
$$

The prediction process starts only after the inference engine has collected a significant number of requests obtaining a DTMC that well represents the client's behaviour. This minimum number of requests is a parameter that can be tuned for each client to meet its specific characteristics.

\subsection{Instantiating Request Parameters}

The service provider serves the received requests and computes their responses ignoring the piggyback parameter appended to them. Before sending the responses back to client, for each request that contains the piggyback parameter the provider invokes the piggybacking engine forwarding to it the following elements: (1) the request including its piggybacking parameter and (2) the computed response. The piggybacking engine is in charge of transforming the predicted endpoint (the piggybacking parameter) to a concrete service request: a valid url with its appropriate parameters. To this end the engine looks up in the RDL file the url structure of the predicted endpoint, extracts the parameters it contains composing a set of parameters $\mathcal{M}$. To instantiate a valid url for the predicted endpoint, the piggybacking engine finds a suitable value for all the elements in $\mathcal{M}$ relying on the following heuristics: 
1. The engine extracts all the parameter values contained in the client request. The engine relies on these extracted values to instantiate the corresponding parameters in $\mathcal{M}$.

2. The engine examines the response to the current request and extracts the parameters specified in the response field as defined in the RDL file (see for example Listing 1.1). The engine relies on these extracted values to instantiate the corresponding parameters in $\mathcal{M}$.

3. The engine considers the most recent past requests of the predicted endpoint and uses their parameter values to instantiate the parameters in $\mathcal{M}$.

4. If by applying the above steps the engine could not instantiate all the parameters in $\mathcal{M}$, the engine relies on their default value if available.

The engine proceeds with the piggybacking process if and only all the parameters in $\mathcal{M}$ have a corresponding value for their instantiation obtained through the heuristics described above. Otherwise the engine aborts the piggybacking process for this request. Let us consider the case in which the client issued the url exemplified in the previous paragraph where the model prediction corresponds to the similar endpoint. Given the structure of the similar endpoint as specified in the RDL file we have that $\mathcal{M}=\{i d$,apikey,limit $\}$. The id as well as the apikey parameters are collected from the url request issued by the client (i.e., id= 77 , apikey $=798)$, while the limit parameter is extracted from previous similar requests issued to the server or using its default value (limit $=5$ ). Considering this second case the predicted request to the similar endpoint is instantiated as:

/movies/77/similar.json?apikey=798\&limit=5

Similarly, let us consider the case in which the client issued a request to the search endpoint where the prediction corresponds to the info endpoint. In this case we have that $\mathcal{M}=\{$ apikey, $i d\}$. The apikey parameter is collected as in the previous case. However, the id parameter is not present in the request issued by the client (the search endpoint does not contain any id parameter). The piggybacking engine collects it from the response to the search request computed by the service provider. Indeed, the engine applies step 2 of the heuristics and relies on the JSONPath expression reported in Listing 1.1 to instantiate the id parameter. This process yields the following url (assuming the result of the search request produced an array in which the first item has id equal to 48):

$$
\text { /movies/48.json?apikey=798 }
$$

As shown by this example, the RDL response field may be used to exploit regularities in the usage profile of the clients to instantiate the predicted url. In this case, we rely on the fact that the first result in the movie returned by the search is the most relevant result and thus the most likely parameter for the predicted info endpoint. If the piggybacking engine successfully instantiates a url corresponding to the predicted endpoint, the provider serves it as if it was a request issued by the client. At this stage the service provider has two responses: the response computed for the request issued by the client and the response computed 
from the url instantiated by the piggybacking engine. The provider sends back to the client a unique response message that encapsulates the second one in a specific field. On the client side the inference engine intercepts the response message sent from the service provider and forwards the first response to the client, while caching internally the response to the predicted endpoint. A subsequent request of the client to the predicted endpoint with the same parameters used by piggybacking engine will result in a cache hit. In such a case, the result is available to the client with zero latency and without exchanging any additional message. The effectiveness of the heuristics described in this section is application specific, and thus the piggybacking engine is open to possible extensions and customisations to fit the specific requirements of applications.

\section{$5 \quad$ Evaluation of Probabilistic Piggybacking}

Efficiency. Let us consider two endpoints $A$ and $B$ offered by a provider and let us consider the case of a client that, after invoking $A$, invokes $B$ with probability to $p_{A B}$. By relying on the maximum likelihood estimator, see Section 4.2. the transition between the states representing $A$ and $B$ will eventually be labelled with probability $p_{A B}$. If $p_{A B}$ is greater than the piggybacking threshold, each response to requests to $A$ is piggybacked with a response to $B$. Thus, the overall probability for a client to experience a cache hit is: $p_{\text {hit }}=p_{A B} \times p_{\text {param }}$, where the first term indicates the probability of having a request to $B$ after a request to $A$, while the second term indicates the probability that requests to $B$ are correctly instantiated by the piggybacking engine. Notice that, for every cache hit, the client and the server exchanged only two messages (request and response), while in the case of a cache miss the client and the sever exchanged four messages (two requests and two responses). Thus the average number of messages exchanged $\left(m_{p}\right)$ can thus be estimated as follows:

$$
m_{p}=p_{\text {hit }} \times 2+\left(1-p_{\text {hit }}\right) \times 4
$$

Since $p_{\text {hit }}$ is a probability $\left(0 \leq p_{\text {hit }} \leq 1\right)$ we have that $m_{p} \leq 4$, while the number of messages exchanged by implementations without piggybacking is four. This indicates that the average number of message exchanged with piggybacking is always less than or equal to the number of messages exchanged by an implementation that does not rely on it and the difference among these two values depends on the probability of cache hits. We measured approximately these results by considering the Flixster movie services. We extended an existing Java client 7 with our inference engine and we produced sequences of invocations to the the similar and to the info endpoint. We also built a proxy of the Flixster movie services to serve these requests relying on our piggybacking engine. We artificially generated the client requests with an increasing value of $p_{\text {info,similar }}$ and a fixed value of $p_{\text {param }}$. Running the client, we measured the average number of messages exchanged with and without the probabilistic piggybacking.

\footnotetext{
${ }^{7}$ JTomato: http://giordano. webfactional. com/?page_id=22
} 


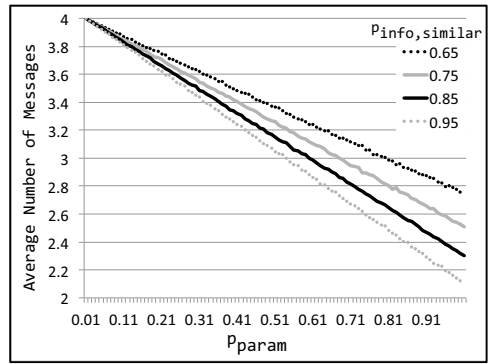

(a) Average Messages.

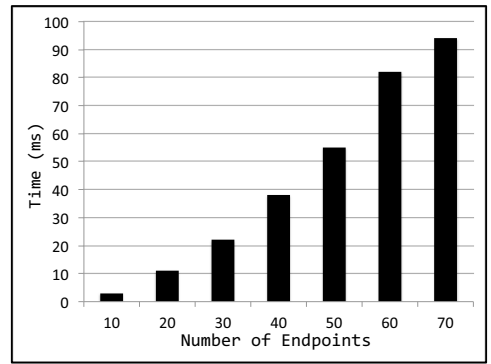

(b) Model Update Overhead.

Fig. 2. Simulation results

If piggybacking is disabled, we measured a constant number of exchanged messages equal to four. Enabling piggybacking - with a piggybacking threshold equal to 0.6 - we obtained an average number of messages always less than or equal to four as illustrated in Figure 2(a) In particular, the average number of messages depends linearly on the parameters $p_{\text {info,similar }}$ and $p_{\text {param }}$ as indicated in Equation 11. In general, the larger their product is (i.e., $p_{\text {hit }}$ ), the more efficient the interaction among the client and the service provider is. In addition to the increased efficiency in terms of exchanged messages, probabilistic piggybacking also brings an additional advantage to the client side in terms of latency. Indeed, each cache hit corresponds to a service invocation issued by the client that can be resolved locally and thus immediately without any network latency. Probabilistic piggybacking introduces an overhead which may be relevant on the client side, in particular in the specific case of mobile clients. To measure this overhead we ran our inference engine on an Android 8 client measuring the average time required by the inference engine to process a service request updating an inferred model of increasing dimension. Figure 2(b) shows that the overhead is negligible. For example the average time required to update a DTMC of 40 states corresponding to a service with 40 endpoints - is only $40 \mathrm{~ms}$. In this evaluation we measured the increased efficiency in terms of exchanged messages and not in terms of generated network traffic. Indeed, in this domain, a real bottleneck is the number of connections opened with clients (see for example the well known C10K problem [10]), which directly depends on the number of service requests invoked by them. Similarly, from the perspective of mobile clients, the metric to be minimised is the number of messages since, because of their mobile nature, each additional message may potentially require a new connection (and thus a new TCP/IP handshake) that is a considerable overhead in terms of radio (i.e., battery) usage and latency. Due to the lack of space we cannot report other relevant scenarios. To support the replicability of results we released our current prototype as an open source artefact 9 .

\footnotetext{
${ }^{8}$ HTC Wildfire, Android 2.1.

9 http://giordano.webfactional. com/?page_id=22
} 
Adaptability to Multiple Usage Profiles. The inferred DTMC captures two distinct usage profiles: (1) the application profile, and (2) the user profile. The application profile refers to patterns in service requests caused by the structure of the client application. Let us consider for example a mobile application that relies on the Flixster movie services. Let us imagine that this mobile application includes a frame that: (1) displays the details of a given movies and (2) lists a set of recommended similar movies. To develop this frame the developers invoke the info endpoint to retrieve the detail of the movie and subsequently the similar endpoint to display the recommended movies. The causal correlation among these two requests is captured by the inferred DTMC as a transition between the states corresponding to these endpoints labelled with an high probability of occurrence (equal to one if the application invokes the info endpoint only in this application frame). In this case the probabilistic piggybacking is extremely effective since the client's behaviour is easily predictable. Indeed, in this case, the correlation between the invocations is hardcoded in the client application. The user profile refers instead to patterns in service requests caused by frequent behaviours of the final users of the client application. For example, as previously exemplified in Section 4.4, given a request to the search endpoint the user may subsequently select - in the majority of cases - the first item in the result set returned by the search. Our solution seamlessly captures also this kind of usage patterns by piggybacking the appropriate response.

Transparency to Clients. Clients typically issue requests to service endpoints using http libraries that are available off the shelf for all the commonly adopted programming languages. The inference engine is an extension of these libraries that issues the requests, handles the responses, infers a DTMC, and caches the responses piggybacked by the provider. From this viewpoint, probabilistic piggybacking is totally transparent to the clients that invoke requests through the inference engine instead of invoking them through the standard http library. Our current implementation of the inference engine consists of an extension of the popular Java http library Apache HttpComponents10.

Complementarity to Caching Techniques. Efficient service provisioning is so crucial in modern service oriented applications that clients always try to optimise the way in which they use services, for example adopting caching mechanisms to reduce the number of interactions with the providers. An additional advantage of the probabilistic piggybacking technique is its complementarity with respect to the traditional caching of requests. Indeed, while caching minimises the interactions with the service provider by exploiting recurrent past service invocations, probabilistic piggybacking minimises the interactions by predicting and anticipating future service requests, similarly to server-side active caches [3]. These techniques are orthogonal and complementary. Indeed, the piggybacked responses may be stored in the same cache used by traditional caching solutions.

${ }^{10}$ http://hc.apache.org 


\section{Related Work}

The problem of inferring and analysing the client behaviours has been address by many approaches for several distinct goals. For example, Liu and V. Kešelj combine the analysis of Web server logs with the contents of the requested Web pages to predict users future requests [13. They capture the content of Web pages by extracting character $\mathrm{N}$-grams that are combined with the data extracted from the log files. Alternatively, Schechter et al. use a tree-based data structure to represent the collection of paths inferred from the log file and predict the next page access [20]. Markov models are the most commonly adopted framework to represent clients interactions. Indeed, such models provide an approximate abstraction of client behaviours that, when applicable in a given domain [4, balance well complexity and expressiveness. Borges and Levene propose a Markov model for representing user navigation sessions inferred from log files and modelled with hypertext probabilistic grammars whose higher probability strings correspond to the users navigation patterns [2]. Sarukkai relies on Markov chains for predicting links and analysing paths by using an inference mechanism similar to the one proposed in this paper [19]. Considering the specific problem of optimising the service provisioning mechanisms we can mention the work by Krashinsky [1] that investigates how to optimise the final critical link between a mobile client and a stationary base station by compressing http request and response messages. Similarly, Tian et al. 21] discuss the benefits obtained by compressing

the messages in XML. Krishnamurthy et al. 12 categorises instead the set of clients communicating with a server in order to optimise the communication for example optimising the interactions with clients characterised by a limited connectivity altering the caching policies. In [14] Papageorgiou et al. analyse the factors that affect the consumption of services by mobile devices in terms of efficiency by comparing different approaches and protocols. Papageorgiou et al. [15] discuss the effectiveness of client side cache for services and mobile clients. Finally, concerning specifically the REST architectural style we can mention the work by Hamad et al. 9] that evaluates the efficiency of REST against SOAP services. Probabilistic piggybacking differs and complements these solutions since it focuses on optimising the service provisioning mechanisms minimising the number of exchanged messages and exploiting a probabilistic approach specifically conceived for mobile clients.

\section{Conclusions and Future Work}

In this paper we discussed a novel technique to optimise the flow of service requests and the client latency. The proposed solution is transparent to the client and complementary to other existing related techniques such as caching. Future work includes a larger validation campaign based on logs of real service invocations and an extension to support services implemented with other architectural styles. 


\section{References}

1. Berglund, A., Boag, S., Chamberlin, D., Fernandez, M.F., Kay, M., Robie, J., Siméon, J.: Xml path language (xpath) 2.0. W3C recommendation, 23 (2007)

2. Borges, J., Levene, M.: Evaluating variable-length markov chain models for analysis of user web navigation sessions. IEEE Transactions on Knowledge and Data Engineering 19(4), 441-452 (2007)

3. Cao, P., Zhang, J., Beach, K.: Active cache: Caching dynamic contents on the web. Distributed Systems Engineering 6(1), 43 (1999)

4. Chierichetti, F., Kumar, R., Raghavan, P., Sarlós, T.: Are web users really markovian? In: WWW. ACM (2012)

5. Chinnici, R., Moreau, J., Ryman, A., Weerawarana, S.: Web services description language version 2.0 part 1: Core language. W3C Recommendation, 26 (2007)

6. DeGroot, M.H., Schervish, M.J.: Probability and Statistics-International Edition. Addison-Wesley Publishing Company, Reading (2001)

7. Fielding, R.T., Gettys, J., Mogul, J., Frystyk, H., Masinter, L., Leach, P., BernersLee, T.: Hypertext transfer protocol-http/1.1 (1999)

8. Fielding, R.T., Taylor, R.N.: Principled design of the modern web architecture. ACM Transactions on Internet Technology (TOIT) 2(2), 115-150 (2002)

9. Hamad, H., Saad, M., Abed, R.: Performance evaluation of restful web services for mobile devices. International Arab Journal of e-Technology (2010)

10. Kegel, D.: The c10k problem (2006), http://www.kegel.com/c10k.html

11. Krashinsky, R.: Efficient web browsing for mobile clients using http compression (2003), http://www.cag.lcs.mit.edu/ ronny/classes/httpcomp.pdf

12. Krishnamurthy, B., Wills, C.E.: Improving web performance by client characterization driven server adaptation. In: Proceedings of the 11th International Conference on World Wide Web, pp. 305-316. ACM (2002)

13. Liu, H., Kešelj, V.: Combined mining of web server logs and web contents for classifying user navigation patterns and predicting users future requests. Data \& Knowledge Engineering 61(2), 304-330 (2007)

14. Papageorgiou, A., Blendin, J., Miede, A., Eckert, J., Steinmetz, R.: Study and comparison of adaptation mechanisms for performance enhancements of mobile web service consumption. In: Services. IEEE (2010)

15. Papageorgiou, A., Schatke, M., Schulte, S., Steinmetz, R.: Enhancing the caching of web service responses on wireless clients. In: 2011 IEEE International Conference on Web Services (ICWS), pp. 9-16. IEEE (2011)

16. Papazoglou, M.P.: Service-oriented computing: Concepts, characteristics and directions. In: Proceedings of the Fourth International Conference on Web Information Systems Engineering, WISE 2003, pp. 3-12. IEEE (2003)

17. Postel, J.: Rfc 793: Transmission control protocol, september 1981 (2003)

18. Ross, S.M.: Stochastic processes. Wiley Series in Probability and Mathematical Statistics, EUA (1983)

19. Sarukkai, R.R.: Link prediction and path analysis using markov chains. Computer Networks 33(1), 377-386 (2000)

20. Schechter, S., Krishnan, M., Smith, M.D.: Using path profiles to predict http requests. Computer Networks and ISDN Systems 30(1), 457-467 (1998)

21. Tian, M., Voigt, T., Naumowicz, T., Ritter, H., Schiller, J.: Performance considerations for mobile web services. Computer Communications 27(11), 1097-1105 (2004)

22. Wilde, E., Pautasso, C.: REST: From Research to Practice. Springer (2011) 